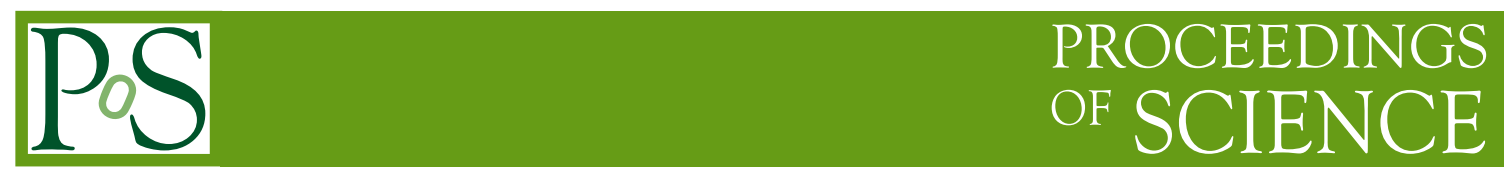

\title{
Single-top production with the POWHEG method
}

\author{
Emanuele $\mathbf{R e}^{* \dagger}$ \\ IPPP, Durham University \\ E-mail: emanuele.re@durham.ac.uk
}

We describe briefly the POWHEG method and present results for single-top s- and t-channel production at hadron colliders.

XVIII International Workshop on Deep-Inelastic Scattering and Related Subjects April 19 -23, 2010

Convitto della Calza, Firenze, Italy

\footnotetext{
* Speaker.

${ }^{\dagger}$ Preprint numbers: IPPP/10/46, DCPT/10/92.
} 


\section{The POWHEG method}

Monte Carlo event generators and NLO QCD calculations are important to give reliable predictions for signals and backgrounds relevant for collider phenomenology. To exploit the advantages of both the approaches it is clear that a method to include NLO QCD corrections to event generators is desirable, especially in view of the amount of data that LHC will collect. The POWHEG method is a prescription to interface NLO calculations with parton shower generators avoiding double counting of emissions, achieving therefore the aforementioned task.

The method was first suggested in ref. [1], and was described in great detail in ref. [2]. It has been applied to several processes at lepton $[3,4]$ and hadron $[5,6,7,8,9,10,11,12,13]$ colliders. Unlike MC@NLO [14], POWHEG produces events with positive weight, and, furthermore, does not depend on the subsequent shower Monte Carlo program: it has been interfaced to HERWIG [15], PYTHIA [16] and HERWIG++ [17]. Up to now all the implementations have been tested against the ones available in $\mathrm{MC} @ \mathrm{NLO}$ : reasonable agreement has been found as well as the reason for the (few) differences encountered (see for example [18] for a recent discussion).

In the POWHEG formalism, the generation of the hardest emission is performed first, according to the distribution given by

$$
d \sigma=\bar{B}\left(\Phi_{B}\right) d \Phi_{B}\left[\Delta_{R}\left(p_{T}^{\min }\right)+\frac{R\left(\Phi_{R}\right)}{B\left(\Phi_{B}\right)} \Delta_{R}\left(k_{T}\left(\Phi_{R}\right)\right) d \Phi_{\mathrm{rad}}\right]
$$

where $B\left(\Phi_{B}\right)$ is the leading order contribution,

$$
\bar{B}\left(\Phi_{B}\right)=B\left(\Phi_{B}\right)+\left[V\left(\Phi_{B}\right)+\int d \Phi_{\operatorname{rad}} R\left(\Phi_{R}\right)\right]
$$

is the NLO differential cross section integrated on the radiation variables while keeping the Born kinematics fixed $\left(V\left(\Phi_{B}\right)\right.$ and $R\left(\Phi_{R}\right)$ stand respectively for the virtual and the real corrections to the Born process), and

$$
\Delta_{R}\left(p_{T}\right)=\exp \left[-\int d \Phi_{\mathrm{rad}} \frac{R\left(\Phi_{R}\right)}{B\left(\Phi_{B}\right)} \theta\left(k_{T}\left(\Phi_{R}\right)-p_{T}\right)\right]
$$

is the POWHEG Sudakov. With $k_{T}\left(\Phi_{R}\right)$ we denote the transverse momentum of the emitted particle. The cancellation of soft and collinear singularities is understood in the expression within the square bracket in eq. (1.2). Partonic events with hardest emission generated according to eq. (1.1) are then showered with a $k_{T}$-veto on following emissions. ${ }^{1}$

Eq. (1.1) is useful to understand easily the main properties of the method. Nevertheless, it is not a trivial task to build a code that takes into account all the technicalities (and the numerical subtleties) hided in eq. (1.1). Although it has been possible to build standalone codes for simple processes, an automated tool (named POWHEG-BOX [19]) was build in order to deal with more complex cases. This program has been already used to implement Higgs-boson production via vector-boson fusion and V + 1jet [20] with POWHEG. Recently, some of the previous implementations have been also included in this package.

\footnotetext{
${ }^{1}$ We refer to $[1,2]$ for all the technicalities that we are neglecting here.
} 


\section{Single-top with POWHEG}

Single-top processes are the ones where only one top quark is produced in the final state. In literature it is customary to categorize the production modes as $s^{-}, t$ - and $W t$-channel, in accordance with the virtuality of the $W$ boson involved in the scattering. Cross sections are smaller than the $t \bar{t}$ pair one, due to their weak nature. This fact, together with the presence of large $\mathrm{W}+$ jet and $t \bar{t}$ backgrounds, makes the single-top observation very challenging, so that this signal has been observed only recently by the CDF and D0 collaborations [21, 22].

Despite of its relative small cross section, single-top production is an important signal for several reasons (for a recent review see for example ref. [23]) and it will be studied thoroughly at the LHC. It is therefore natural to provide the experimental community with a tool that performs the merging between NLO and parton showers for this process. This has been accomplished some time ago within the MC@NLO framework [24, 25], whereas a POWHEG implementation for $s$ - and $t$-channel has been completed more recently in a standalone code, and then also included in the POWHEG-BOX package. ${ }^{2}$ An implementation for $W t$-channel is also under way.

In figures 1 and 2 we present some typical distributions for single-top $t$-channel $\bar{t}$ and $t$ production at the LHC respectively. Results have been obtained choosing $\sqrt{S}=7 \mathrm{TeV}, m_{t}=173.1$ $\mathrm{GeV}$ and $m_{W}=80.4 \mathrm{GeV}$. As PDF's we used the CTEQ6M set. Plots have been obtained including the top-quark semileptonic decay, but removing the branching ratio.

The very good agreement with the MC@NLO results shows that the method works properly. In the following we discuss in more detail the differences with respect to PYTHIA predictions.

We start by recalling that PYTHIA results have been rescaled to the POWHEG (and MC@NLO) ones, since their normalization is only LO accurate.

Concerning the $p_{T}$ spectrum of the hardest $\bar{b}$-flavoured hadron ( $b$-flavoured for anti-top production), the difference is due to the absence of matrix-element corrections in PYTHIA: the $g \rightarrow b \bar{b}$ splitting (typical of $t$-channel single-top) is accurate only in the collinear limit (being it performed by the shower) and therefore the high- $p_{T}$ tail of PYTHIA result shows a lack of events.

In the plot showing the angular correlation between the direction of the charged lepton coming from the top decay and the spectator jet (i.e. the hardest non $b$-flavoured jet), ${ }^{3}$ POWHEG and $\mathrm{MC} @ N L O$ are in good agreement but differ remarkably with PYTHIA. This distribution measures spin-correlation effects between the production and the decay process [27]. To take into account these effects, in POWHEG the top decay is generated with a procedure similar to the one used in MC@NLO [26]. Instead in PYTHIA top decay-products are generated uniformly and the corresponding angular distribution results flat. ${ }^{4}$

\section{Acknowledgements}

Results presented in this proceeding have been obtained in collaboration with S. Alioli, P. Nason and C. Oleari. The author wants also to acknowledge G. Corcella for the invitation to the conference and the opportunity to give the talk remotely.

\footnotetext{
${ }^{2}$ Results presented in the following have been obtained with the POWHEG-BOX package.

${ }^{3}$ Here we use the spectator basis [27] and we plot the angle as seen in the top-quark rest frame.

${ }^{4}$ We notice that the damping at high values of $\cos \theta$ is due to the cuts applied.
} 

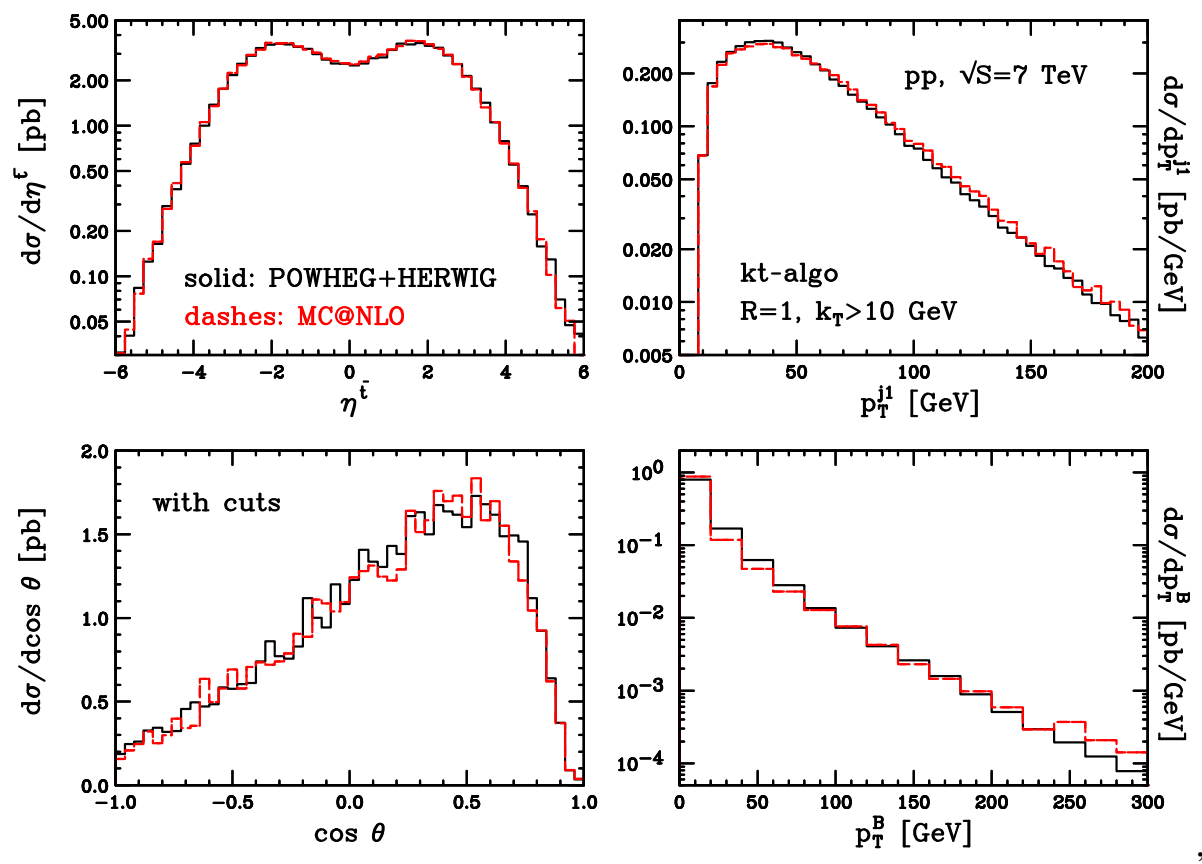

Figure 1: Comparisons between POWHEG and MC@NLO for $t$-channel single-top $\bar{t}$ production at the LHC. Cuts used for angular correlation are: $\left|\eta_{b}\right|<2.5, p_{T}^{b}>50 \mathrm{GeV}, 2.5<\left|\eta_{s j}\right|<5.0, p_{T}^{s j}>30 \mathrm{GeV},\left|\eta_{\ell}\right|<2.5$, $p_{T}^{\ell}>30 \mathrm{GeV}, E_{T}^{\text {miss }}>20 \mathrm{GeV}$. With $s j$ we denote the spectator jet.
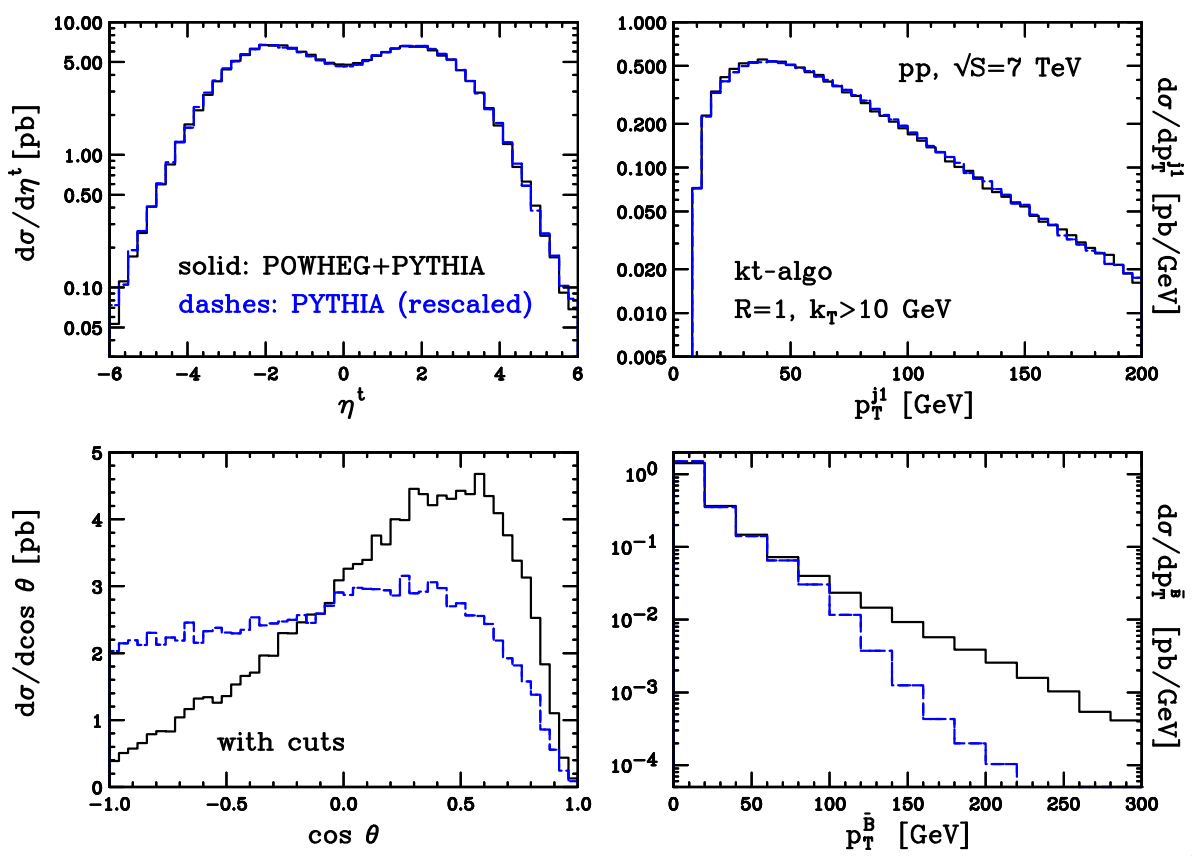

Figure 2: Comparisons between POWHEG and PYTHIA for $t$-channel single-top $t$ production at the LHC. For angular correlation the same cuts as above have been used. 


\section{References}

[1] P. Nason, JHEP 0411, 040 (2004) [arXiv:hep-ph/0409146].

[2] S. Frixione, P. Nason and C. Oleari, JHEP 0711, 070 (2007) [arXiv:0709.2092 [hep-ph]].

[3] O. Latunde-Dada, S. Gieseke and B. Webber, JHEP 0702, 051 (2007) [arXiv:hep-ph/0612281].

[4] O. Latunde-Dada, Eur. Phys. J. C 58, 543 (2008) [arXiv:0806.4560 [hep-ph]].

[5] P. Nason and G. Ridolfi, JHEP 0608, 077 (2006) [arXiv:hep-ph/0606275].

[6] S. Frixione, P. Nason and G. Ridolfi, JHEP 0709, 126 (2007) [arXiv:0707.3088 [hep-ph]].

[7] S. Alioli, P. Nason, C. Oleari and E. Re, JHEP 0807, 060 (2008) [arXiv:0805.4802 [hep-ph]].

[8] K. Hamilton, P. Richardson and J. Tully, JHEP 0810, 015 (2008) [arXiv:0806.0290 [hep-ph]].

[9] A. Papaefstathiou and O. Latunde-Dada, JHEP 0907, 044 (2009) [arXiv:0901.3685 [hep-ph]].

[10] S. Alioli, P. Nason, C. Oleari and E. Re, JHEP 0904, 002 (2009) [arXiv:0812.0578 [hep-ph]].

[11] K. Hamilton, P. Richardson and J. Tully, JHEP 0904, 116 (2009) [arXiv:0903.4345 [hep-ph]].

[12] S. Alioli, P. Nason, C. Oleari and E. Re, JHEP 0909, 111 (2009) [arXiv:0907.4076 [hep-ph]].

[13] P. Nason and C. Oleari, JHEP 1002, 037 (2010) [arXiv:0911.5299 [hep-ph]].

[14] S. Frixione and B. R. Webber, JHEP 0206, 029 (2002) [arXiv:hep-ph/0204244].

[15] G. Corcella et al., JHEP 0101, 010 (2001) [arXiv:hep-ph/0011363].

[16] T. Sjostrand, S. Mrenna and P. Z. Skands, JHEP 0605, 026 (2006) [arXiv:hep-ph/0603175].

[17] M. Bahr et al., Eur. Phys. J. C 58, 639 (2008) [arXiv:0803.0883 [hep-ph]].

[18] P. Nason, arXiv:1001.2747 [Unknown].

[19] S. Alioli, P. Nason, C. Oleari and E. Re, JHEP 1006, 043 (2010) [arXiv:1002.2581 [hep-ph]].

[20] S. Alioli, P. Nason, C. Oleari and E. Re. To appear soon.

[21] V. M. Abazov et al. [D0 Collaboration], Phys. Lett. B 682, 363 (2010) [arXiv:0907.4259 [hep-ex]].

[22] T. Aaltonen et al. [CDF Collaboration], Phys. Rev. Lett. 103 (2009) 092002 [arXiv:0903.0885 [hep-ex]].

[23] W. Bernreuther, J. Phys. G 35, 083001 (2008) [arXiv:0805.1333 [hep-ph]].

[24] S. Frixione, E. Laenen, P. Motylinski and B. R. Webber, JHEP 0603, 092 (2006) [arXiv:hep-ph/0512250].

[25] S. Frixione, E. Laenen, P. Motylinski, B. R. Webber and C. D. White, JHEP 0807, 029 (2008) [arXiv:0805.3067 [hep-ph]].

[26] S. Frixione, E. Laenen, P. Motylinski and B. R. Webber, JHEP 0704, 081 (2007) [arXiv:hep-ph/0702198].

[27] G. Mahlon and S. J. Parke, Phys. Lett. B 476, 323 (2000) [arXiv:hep-ph/9912458]. 